\title{
The osteoporosis treatment gap in patients at risk of fracture in European primary care: a multi-country cross-sectional observational study
}

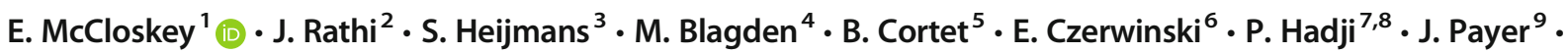

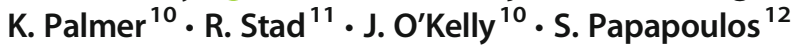

Received: 22 March 2020 / Accepted: 21 July 2020 / Published online: 23 August 2020

(C) The Author(s) 2020

\begin{abstract}
Summary This study in 8 countries across Europe found that about $75 \%$ of elderly women seen in primary care who were at high risk of osteoporosis-related fractures were not receiving appropriate medication. Lack of osteoporosis diagnosis appeared to be an important contributing factor.

Introduction Treatment rates in osteoporosis are documented to be low. We wished to assess the osteoporosis treatment gap in women $\geq 70$ years in routine primary care across Europe.

Methods This cross-sectional observational study in 8 European countries collected data from women 70 years or older visiting their general practitioner. The primary outcome was treatment gap: the proportion who were not receiving any osteoporosis medication among those at increased risk of fragility fracture (using history of fracture, 10-year probability of fracture above country-specific Fracture Risk Assessment Tool [FRAX] thresholds, T-score $\leq-2.5$ ).

Results Median 10-year probability of fracture (without bone mineral density [BMD]) for the 3798 enrolled patients was $7.2 \%$ (hip) and 16.6\% (major osteoporotic). Overall, 2077 women (55\%) met one or more definitions for increased risk of fragility fracture: 1200 had a prior fracture, 1814 exceeded the FRAX threshold, and 318 had a T-score $\leq-2.5$ (only 944 received a dualenergy x-ray absorptiometry [DXA] scan). In those at increased fracture risk, the median 10-year probability of hip and major osteoporotic fracture was $11.2 \%$ and $22.8 \%$, vs $4.1 \%$ and $11.5 \%$ in those deemed not at risk. An osteoporosis diagnosis was recorded in 804 patients $(21.2 \%)$; most $(79.7 \%)$ of these were at increased fracture risk. The treatment gap was $74.6 \%$, varying from $53 \%$ in Ireland to $91 \%$ in Germany. Patients with an osteoporosis diagnosis were found to have a lower treatment gap than those without a diagnosis, with an absolute reduction of $63 \%$.

Conclusions There is a large treatment gap in women aged $\geq 70$ years at increased risk of fragility fracture in routine primary care across Europe. The gap appears to be related to a low rate of osteoporosis diagnosis.
\end{abstract}

Keywords Fragility fracture $\cdot$ Observational study $\cdot$ Osteoporosis $\cdot$ Primary care $\cdot$ Treatment gap

Electronic supplementary material The online version of this article (https://doi.org/10.1007/s00198-020-05557-z) contains supplementary material, which is available to authorized users.

E. McCloskey

E.V.McCloskey@sheffield.ac.uk

1 Centre for Metabolic Bone Diseases, University of Sheffield, Sheffield, UK

2 Carrig Medical Centre, Cork, Ireland

3 ResearchLink, Linkebeek, Belgium

4 Ashgate Medical Practice, Chesterfield, UK

5 Department of Rheumatology and EA 4490, University-Hospital of Lille, Lille, France
6 Department of Bone and Joint Diseases, FHS, Jagiellonian University Medical College, Kopernika 32, 31-501 Krakow, Poland

7 Frankfurt Center of Bone Health, Frankfurt, Germany

8 Philipps-University of Marburg, Marburg, Germany

9 Faculty of Medicine, 5th Department of Internal Medicine in University Hospital Bratislava, Comenius University, Bratislava, Slovakia

10 Amgen Ltd, Uxbridge, UK

11 Amgen Europe GmbH, Rotkreuz, Switzerland

12 Leiden University Medical Center, Leiden, Netherlands 


\section{Introduction}

Over 8.9 million osteoporotic or fragility fractures occur every year across the globe, more than a third of which occur in Europe $[1,2]$. In 2010, 3.5 million new fragility fractures (FFs) in 22 million women aged over 50 were estimated to have occurred across the EU; and FFs are predicted to increase due to changing demographics [3]. It has been suggested that approximately $51 \%$ of the hip fractures that occur globally are potentially preventable [4]. Hence, disease awareness, early diagnosis, prevention, and appropriate treatment of fractures are critical in osteoporosis (OP) management. However, the diagnosis of OP frequently occurs only after a fracture, and even then treatment rates can be low [4].

General practitioners (GPs) play a critical role in OP management. To begin with, they are well positioned to assess risk factors in the general population [5]. For example, in a crosssectional survey on women 45 years or older in the French general population, the diagnosis of $\mathrm{OP}$ was most commonly made by a GP (approximately $45 \%$ of diagnoses [6]); and in a single OP clinic in the UK, $94 \%$ of the referrals for bone mineral density (BMD) scans over the course of 1 year had been made by GPs [7]. For patients who experience OPrelated fractures, a cost burden is also placed on primary care. A study of UK databases reported that primary care costs increased significantly in the first 2 years after hip fracture [8]. Coordinator-based fracture liaison services for secondary prevention also usually require the GP's involvement as part of a multi-disciplinary team for treating patients and monitoring their progress $[9,10]$.

Diagnostic methods and fracture risk assessment have improved in recent years. However, despite new interventions to reduce fracture risk and new clinical practice guidelines, only a small fraction of eligible women receive treatment for OP, implying a high treatment gap [11]. It was estimated that 10.6 out of the 18.4 million women in the EU who exceeded the threshold risk for FF were not treated, representing a treatment gap of 57\% [3]. This is likely to be an underestimate, as the analysis assumed that all OP drug usage was targeted appropriately at individuals at high risk. In the absence of recent real-world data, we designed this study to specifically focus on assessing the treatment gap in a large sample of women $\geq$ 70 years in Europe who were routinely visiting their GPs.

\section{Methods}

\section{Study design}

This cross-sectional, multicenter, multi-country observational study included 153 GP sites across 8 countries: Belgium, France, Germany, Ireland, Poland, Slovakia, Switzerland, and the UK. Countries were included based on regulatory requirements and feasibility. GP offices were selected based on interest in participation as a study site, and willingness and ability to comply with the protocol and data entry conventions. Community-dwelling women 70 years or older who spontaneously visited their GP were invited to participate, and, following informed consent, completed a short selfreported questionnaire.

The primary objective was to assess the proportion of women $\geq 70$ years who were at increased risk of FF (see following) and not receiving any OP medication. Increased risk of FF was defined by the presence of at least one of the following three base criteria:

1. History of fracture after the age of 50 (hip, spine, wrist, or other OP-related fractures)

2. Increased 10-year probability of both hip and major osteoporotic fracture (calculated using Fracture Risk Assessment Tool [FRAX] without BMD above countryspecific threshold (Table S1), using an approach similar to that recommended by the National Osteoporosis Guidance Group, UK [9])

3. BMD T-score $\leq-2.5$ for lumbar spine, total hip, or femoral neck.

Key secondary objectives included the description of patient characteristics among those at increased risk of FF or not, and a description of the types of OP medications used.

Specific osteoporosis medications were assessed in the overall population, according to fracture history and according to current or previous use (within the last 10 years). Line of treatment was assessed among current users: no previously documented osteoporosis treatment indicated first-line use, and one type of previous osteoporosis medication indicated second-line use.

Sensitivity analyses were conducted using alternative definitions of increased risk of FF based on FRAX without BMD (criterion 2) and involved increasing or decreasing the major osteoporotic fracture and hip fracture thresholds by absolute values of $5 \%$ and $2 \%$, respectively. A further analysis examined the same threshold as in the primary analysis, but with the FRAX probabilities calculated with the inclusion of BMD, where available.

\section{Data collection}

The following variables were collected from the patient's existing medical records: demographics (age, gender, country); weight; height; body mass index (BMI); reason for consultation; smoking status; alcohol consumption; parental history of hip fracture; fracture history; falls in the past year; any known OP diagnosis; BMD results from last dual-energy $\mathrm{x}$ ray absorptiometry (DXA) scan, if available; use of glucocorticoids; comorbidities (rheumatoid arthritis [RA], diabetes, 
hypertension, osteoarthritis, and chronic obstructive pulmonary disease $[\mathrm{COPD}]$ ) and associated treatments; OP medications used within the last 10 years (selective estrogen receptor modulators [SERMs], oral bisphosphonates [BPs], parenteral BPs, strontium ranelate, parathyroid hormone [PTH], antireceptor activator of nuclear factor kappa-B ligand [antiRANKL]/denosumab; use of vitamin D/calcium supplements).

Questionnaires were administered at the patient's single GP visit, which asked for information on the patient's age, height, weight, alcohol intake, parental hip fracture, history of fracture after age 50, and falls in the last year. Where there were discrepancies between medical records and the questionnaires, data from medical records were used.

\section{Study size}

No formal hypothesis was tested. A sample size of 4000 (500/ country) was expected to estimate the primary outcome for each participating country with sufficient precision, assuming that $40 \%$ of women $\geq 70$ years of age would be at increased risk of fracture and at least $50 \%$ of these would not be receiving an OP medication.

\section{Statistical analysis}

All analyses were descriptive. No statistical inference or imputations of missing data were planned. Analyses of the primary outcome were based on the primary analysis subset, i.e., all enrolled patients who were at increased risk of FF. Where applicable, secondary analyses were done on the full analysis set (FAS), consisting of all enrolled patients. Sensitivity analyses of the primary and key secondary outcomes were performed using different definitions of increased risk of FF (described earlier).

\section{Results}

\section{Description of overall study population}

Data were collected between 28 March 2018 and 26 October 2018. A total of 3798 patients (median age, 77 years) were enrolled. In each country, approximately 500 patients were enrolled across 18 to 25 sites (Table 1), except for Switzerland ( $n=205$ across 6 sites). The largest number was enrolled in France $(n=543)$. The most common reason for consultation was "follow-up to known disease" (52.1\%), followed by "new symptoms/complaints" (21.7\%), "medication refill" (20.6\%), and "other" (5.6\%). The median 10-year probability of hip fracture was $7.2 \%$, and of major osteoporotic fracture, $16.6 \%$ (both calculated without BMD; Table 1).
Table 1 Overall patient characteristics

\begin{tabular}{ll}
\hline Parameter & Descriptive statistics \\
\hline Country of recruitment $(N)$ & 505 \\
Belgium & 543 \\
France & 506 \\
Germany & 500 \\
Ireland & 505 \\
Poland & 534 \\
Slovakia & 205 \\
Switzerland & 500 \\
UK & $77.0(73.0,82.0)$ \\
Age, median (Q1, Q3), years & $26.9(23.9,30.5)$ \\
BMI, median $(\mathrm{Q} 1, \mathrm{Q} 3), \mathrm{kg} / \mathrm{m}^{2}$ & $804(21.2)$ \\
Known OP diagnosis, $n(\%)$ & $1978(52.1)$ \\
Reason for consultation, $n(\%)$ & $782(20.6)$ \\
Follow-up to known disease & $824(21.7)$ \\
Medication refill & $214(5.6)$ \\
New symptoms/complaints & $7.2(4.1,11.9)$ \\
Other & $16.6(11.5,23.9)$ \\
10-year fracture probability without BMD, median $(\mathrm{Q} 1, \mathrm{Q} 3), \%$ \\
Hip fracture
\end{tabular}

$B M D$, bone mineral density; $B M I$, body mass index; $O P$, osteoporosis; $Q$, quartile

Patient recruitment and classification by fracture risk, OP diagnosis, and whether treated with OP medication or not are shown in Fig. 1. Of all the enrolled patients, 2077 (55\%) were deemed to be at increased risk of FF using the base criteria for fracture risk. Of the total population, 804 patients $(21.2 \%)$ had an OP diagnosis identified in their medical records, and the majority of these $(641,79.7 \%)$ were in the increased fracture risk group. Importantly, BMD results were available in 944 women only (24.9\% of the cohort), with BMD results for lumbar spine available in 908 women $(23.9 \%$ of the whole cohort), for the total hip in 747 women $(19.6 \%$ of the whole cohort), and for the femoral neck in 727 (19.1\% of the whole cohort). Thus, the number classified at high risk based on BMD (T-score $\leq 2.5$ ) was derived from a smaller subset of the cohort.

\section{Prevalence of increased fracture risk and treatment gap}

Those at increased risk of FF were older than those not at increased risk (median 80.0 years vs. 75.0 years; Table 2). They also had a lower median BMI and, as expected, a higher prevalence of risk factors associated with increased fracture risk apart from current smoking and alcohol exposure. Overall, 3361 patients $(88.5 \%)$ reported at least one comorbidity, with similar proportions in patients with and without 


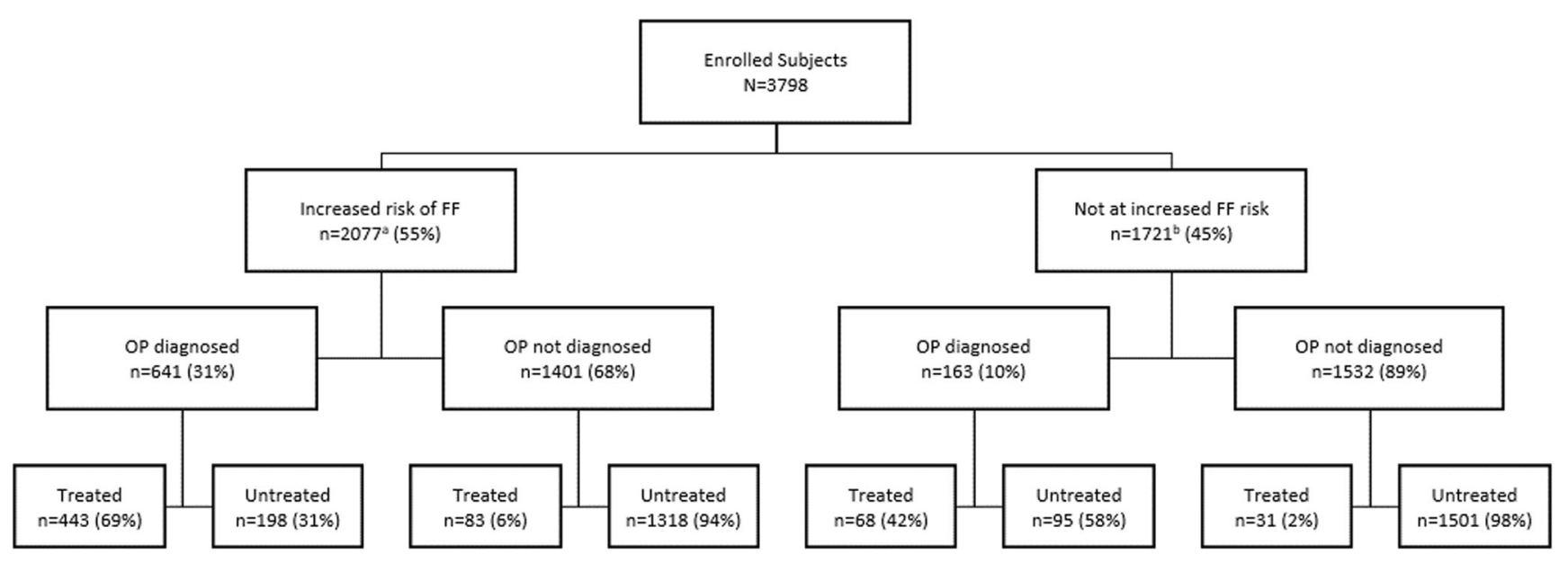

Fig. 1 Patient flowchart (adapted from [12]). FF, fragility fracture; OP, osteoporosis. ${ }^{a}$ Unknown OP diagnosis in 35 patients at increased risk of fracture ( 1 treated, 34 untreated). ${ }^{b}$ Unknown OP diagnosis in 26 patients not at increased risk of fracture (all untreated)

increased risk of FF. The most common comorbidities overall were hypertension $(2840,74.8 \%)$, osteoarthritis $(1702$, $44.8 \%$ ), and diabetes $(953,25.1 \%$; data not shown). The median FRAX 10-year probabilities of a major osteoporotic fracture and hip fracture (calculated without BMD) were 1.98-fold and 2.73-fold higher, respectively, than those not an increased risk (22.8 vs. 11.5 and 11.2 vs. 4.1 , respectively).

For the primary outcome of treatment gap in patients at increased risk of FF, 1550 (74.6\%) were not receiving OP medication (Fig. 2). Across countries, the treatment gap varied from 53\% in Ireland to $91 \%$ in Germany (Online Resource Figure S1).

The treatment gap was notably different depending on whether a diagnosis of OP was recorded. Thus, in women with increased risk and a diagnosis of OP, the treatment gap was only $30.9 \%$, while in those with increased risk and no OP diagnosis, the gap was $94.1 \%$. A diagnosis was therefore associated with an absolute reduction in treatment gap of $63 \%$ (Fig. 2). An ad hoc analysis of the subset of patients who received a DXA scan showed that 54\% (508/944) were not treated; and an analysis of the subset of patients who had a recorded T-score showed that $21 \%$ (66/318) of those with a Tscore $\leq-2.5$ were not treated.

A diagnosis of OP also influenced prescribing in those deemed not to be at increased risk of fracture (Fig. 1). Treatment was only reported in $6 \%$ of those not at increased risk, but this ranged from $2.0 \%$ in those without a diagnosis of OP to $42 \%$ in those with a diagnosis. Regardless of risk of FF, nearly all $(96.1 \%)$ of those without an OP diagnosis did not receive OP medication (Fig. 1).

The reasons for being classified as being at increased risk of FF are shown in Table 3. Of the 2077 women, 1200 $(57.8 \%)$ qualified because they had experienced a prior fracture. Of these, $1020(85 \%)$ also exceeded the country-specific FRAX threshold. A further 794 women were classified because their fracture probability exceeded the country-specific
FRAX threshold. The fracture risk of these patients was similar to that observed in patients reporting a history of prior fracture (Table 3). The fracture risk in those identified by FRAX probability, whether alone or in combination with other criteria, was consistently higher than in those identified without considering fracture probability. Only a small proportion of patients met the definition for increased risk of FF by $\mathrm{T}$-score only (4.0\%), reflecting the fact that BMD results were only available in a small subset $(24.9 \%)$ of the cohort. Of the 944 women with BMD recorded, 318 (33.7\%) had a T-score of $\leq-2.5$ at one of the 3 skeletal sites.

\section{OP medication use}

Overall, 626 patients $(16.5 \%)$ had received or were receiving OP medication, of whom $422(67.4 \%)$ were currently using OP medication and 204 (32.6\%) had used it previously. The most common OP medications used (Table 4) were oral BPs (67.4\%), denosumab (29.9\%), and parenteral BPs (13.6\%). Patients with a history of spine fracture received an OP medication more frequently than those with a history of hip fracture $(57.9 \%$ vs. $35.9 \%)$.

Oral BP constituted the majority of first-line OP medication (58.3\%), followed by denosumab (28.3\%) and parenteral BP $(8.4 \%)$. Denosumab was the most common second-line OP medication (76.3\%), followed by parenteral BP (10.0\%) and oral BP $(7.5 \%)$.

Most (90.1\%) OP medication users also received vitamin D/calcium supplements. $30.5 \%$ of the patients overall received vitamin $\mathrm{D} /$ calcium supplements alone (data not shown).

\section{Subgroup and sensitivity analyses}

Increasing or decreasing the FRAX-based thresholds (as described in the "Methods" section) only had a relatively small 
Table 2 Characteristics, OP diagnosis, comorbidities, clinical risk factors, and 10-year fracture probability in patients with increased risk of fragility fracture vs. those without increased risk

\begin{tabular}{|c|c|c|}
\hline Parameter & $\begin{array}{l}\text { With increased risk of fragility fracture } \\
(N=2077)\end{array}$ & $\begin{array}{l}\text { Without increased risk of fragility fracture }{ }^{\mathrm{a}, \mathrm{b}} \\
(N=1721)\end{array}$ \\
\hline Age, median (Q1, Q3), years & $80.0(75.0,84.0)$ & $75.0(72.0,78.0)$ \\
\hline BMI, median (Q1, Q3), kg/m² & $25.7(22.9,29.3)$ & $28.6(25.5,32.1)$ \\
\hline Known OP diagnosis, $n(\%)$ & $641(30.9)$ & $163(9.5)$ \\
\hline At least one comorbidity, $n(\%)$ & $1854(89.3)$ & 1507 (87.6) \\
\hline \multicolumn{3}{|l|}{ Clinical risk factors for fragility fracture, $n(\%)$} \\
\hline Previous fracture & $1200(57.8)$ & $0(0.0)$ \\
\hline Hip & $145(7.0)$ & $0(0.0)$ \\
\hline Spine & $178(8.6)$ & $0(0.0)$ \\
\hline Wrist & $435(20.1)$ & $0(0.0)$ \\
\hline Other (except skull, finger, and toe fractures) & $634(30.5)$ & $0(0.0)$ \\
\hline Parental hip fracture & $302(14.5)$ & $64(3.7)$ \\
\hline Current smoker & $132(6.4)$ & $99(5.8)$ \\
\hline Glucocorticoid use & $157(7.6)$ & $19(1.1)$ \\
\hline Rheumatoid arthritis & $130(6.2)$ & $21(1.2)$ \\
\hline Alcohol ( $\geq 3$ units per day) & $32(1.5)$ & $18(1.0)$ \\
\hline Femoral neck T-score, median (Q1, Q3) $[n]$ & $-1.8(-2.5,-1.1)[518]$ & $-1.0(-1.7,-0.4)[209]$ \\
\hline \multicolumn{3}{|c|}{ 10-year fracture probability without BMD, median (Q1, Q3), \% } \\
\hline Hip fracture & $11.2(7.6,16.2)$ & $4.1(2.8,6.2)$ \\
\hline Major osteoporotic fracture & $22.8(18.0,29.6)$ & $11.5(8.9,14.7)$ \\
\hline
\end{tabular}

$B M D$, bone mineral density; $B M I$, body mass index; $F F$, fragility fracture; $O P$, osteoporosis

${ }^{a}$ A patient will be considered to be at increased risk of fragility fracture if $\geq 1$ of the 3 following criteria are met: (1) had a history of fracture; (2) 10-year probability of hip fracture without BMD > country-specific threshold and 10-year probability of major osteoporotic fracture without BMD > countryspecific threshold; (3) BMD T-score $\leq-2.5$ for any of lumbar spine/total hip/femoral neck

${ }^{\mathrm{b}}$ Number of patients enrolled in full analysis set. Percentages based on total number of patients in respective increased risk of fragility fracture category using the base definition

effect on the proportion of the population identified at increased risk. The proportion ranged from 52.3 to $57.7 \%$ for the higher and lower thresholds, respectively (Online Resource Table S2). This also had little impact on the estimates of the treatment gap, with values being $74.0 \%$ and $75.7 \%$, respectively (Online Resource Table S3).

Since there were differences in speed of recruitment across centers, we compared the baseline characteristics of the first $50 \%$ of patients enrolled to the next $50 \%$ to see if biases had gradually influenced the types of patients recruited. Reassuringly, the characteristics were remarkably similar including age, reasons for consultation, and OP diagnosis (Online Resource Table S4), as well as for comorbidities (Online Resource Table S5) and fracture history (Online Resource Table S6).

\section{Discussion}

Our study found a large treatment gap for women aged $\geq 70$ years at increased risk of $\mathrm{FF}$ who were in routine primary care across 8 countries in Europe. Overall, just over half were found to be at increased risk of FF, of whom three-quarters were not receiving any OP medication. Even among patients with prior spine or hip fractures, the treatment gap was high, with $42 \%$ and $64 \%$, respectively, not receiving OP treatment. While the treatment gap varied, it was above $50 \%$ in all 8 countries. The treatment gap in patients with prior hip fracture was lowest in Ireland (43\%), Belgium (46\%), and the UK $(55 \%)$, and highest in Switzerland (88\%) and Germany (94\%). We observed that a diagnosis of OP has a substantial impact; the treatment gap was much smaller in those with an OP diagnosis ( $30.9 \%$ vs. $74.6 \%$ in the overall population) and much larger in those without an OP diagnosis $(94.1 \%)$. The treatment gap therefore appears to be largely related to a gap in diagnosis or awareness.

The large treatment gap reported here is consistent with previous studies. For example, treatment gaps of 55-68\% have been reported for Europe using different methodologies $[1,3,13]$. Even in the presence of a prior fracture, treatment rates have been reported to be low for secondary prevention [2] and have also been shown to be declining over time [3]. 
Fig. 2 OP treatment gap for overall patients and by $\mathrm{OP}$ diagnosis (adapted from [12]). CI, confidence interval; $\mathrm{FF}$, fragility fracture; OP, osteoporosis. $N$ represents the number of patients at increased risk of fragility fracture using the base definition. Thirty-five patients had an unknown diagnosis of $\mathrm{OP}$

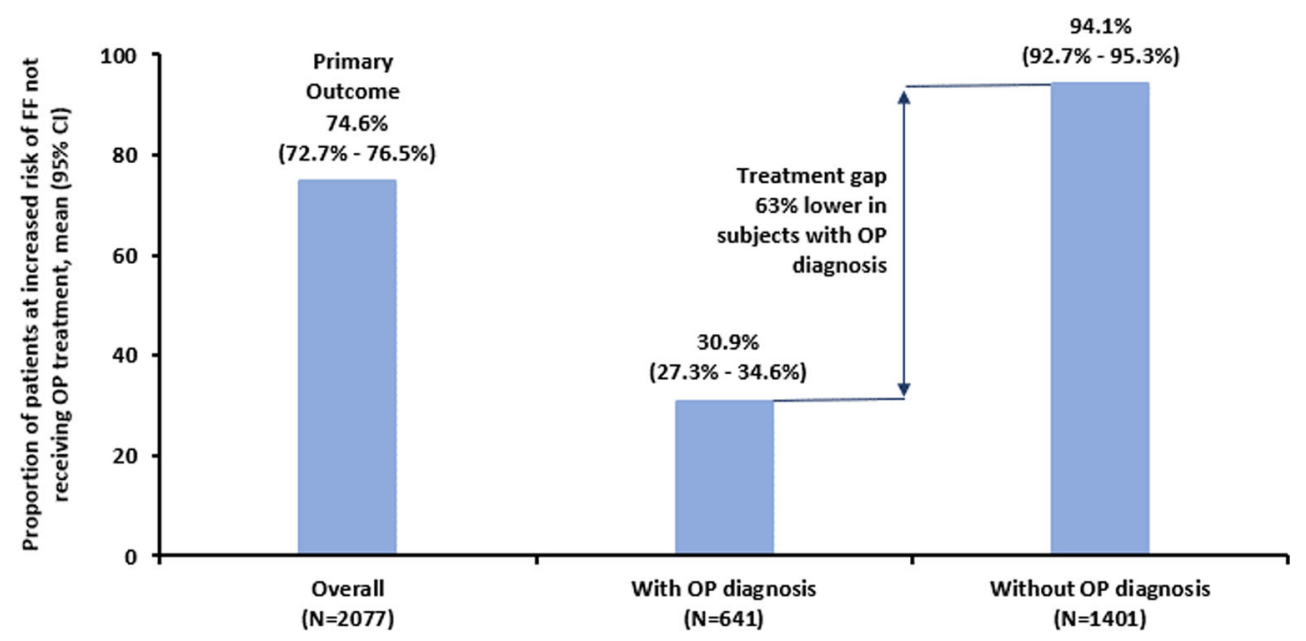

Across the EU, less than $20 \%$ of patients with FFs were reported to receive treatment within the year following fracture [1]. This treatment gap has also been reported in the elderly, for whom the consequences of untreated OP are more severe. Treatment gap was between 48 and 68\% in Swedish women aged over 70 , with as few as $10 \%$ of elderly women with FFs receiving any OP therapy [11]. Wide intercountry variability in treatment uptake in women with higher risk of osteoporotic fractures has also been reported in other studies [1, 3], and could be due to different availability and reimbursement of medications and differences in fracture risk. Fracture registries may play an important role in improving access to treatment: Ireland and the UK have national hip fracture registries and had a relatively low treatment gap compared with other countries. In all of these countries, treatment guidelines suggest a case-finding approach for patient identification, but there is variation in terms of which risk factors are acknowledged, how fracture risk should be assessed, how BMD measurements should be used, and the thresholds for treatment initiation. Notwithstanding the wide availability of national guidelines, it is clear that their recommendations are not always followed.

We observed that a diagnosis of OP has a substantial impact on the treatment gap; in women at high risk, the gap was much smaller in those with an OP diagnosis than in those without (30.9\% vs. $94.1 \%$, respectively). A similarly reduced treatment gap in those with a diagnosis of OP was reported in the GLOW study [13]. A diagnosis of OP was therefore found

Table 3 Proportion of patients at increased risk of fragility fracture and 10-year fracture probability, according to fracture risk criteria

\begin{tabular}{llll}
\hline Parameter & $n(\%)$ & \multicolumn{1}{l}{10 -year fracture probability without BMD, median (Q1, Q3), \% } \\
\cline { 2 - 4 } & & Hip fracture & Major osteoporotic fracture \\
\hline $\begin{array}{l}\text { Number of patients with increased risk of fragility fracture } \\
\text { Meeting 1 of the 3 criteria }\end{array}$ & $2077(100.0)$ & $7.2(4.1,11.9)$ & $16.6(11.5,23.9)$ \\
Prior fracture only & $168(8.1)$ & $4.4(3.3,5.6)$ & $16.2(12.7,17.9)$ \\
FRAX threshold only & $733(35.3)$ & $11.5(8.9,15.8)$ & $21.1(17.6,25.8)$ \\
T-score only & $83(4.0)$ & $5.8(4.1,7.7)$ & $13.6(11.0,16.5)$ \\
Meeting 2 of the 3 criteria & & & $26.7(21.9,32.3)$ \\
Prior fracture and FRAX threshold & $858(41.3)$ & $12.3(8.7,17.4)$ & $17.7(16.4,18.8)$ \\
Prior fracture and T-score & $12(0.6)$ & $5.4(4.0,5.9)$ & $23.2(21.1,27.6)$ \\
FRAX threshold and T-score & $61(2.9)$ & $13.9(11.8,18.9)$ & $30.6(25.1,39.1)$
\end{tabular}

$B M D$, bone mineral density; FRAX, Fracture Risk Assessment Tool; $Q$, quartile

${ }^{\text {a }}$ BMD results were only available in subset $(24.9 \%)$ of the cohort

A patient was considered to be at increased risk of fragility fracture if $\geq 1$ of the 3 following criteria were met: (1) had a history of fracture; (2) 10-year probability of hip fracture without BMD $>$ country-specific threshold and 10-year probability of major osteoporotic fracture without BMD $>$ countryspecific threshold; (3) BMD T-score $\leq-2.5$ for any of lumbar spine/total hip/femoral neck

Groups are mutually exclusive (i.e., patients are only counted once with respect to how they met the criteria)

Percentages based on number of patients enrolled who are at increased risk of fragility fracture using the base definition 
Table 4 OP medication use in the overall population

\begin{tabular}{|c|c|c|c|c|c|c|c|c|}
\hline \multirow[t]{2}{*}{ OP medication type, $n(\%)$} & \multirow[t]{2}{*}{ Overall $(N=3798)$} & \multicolumn{2}{|l|}{ Fracture history } & \multicolumn{2}{|c|}{$\begin{array}{l}\text { OP medication use }(n= \\
626)\end{array}$} & \multicolumn{3}{|c|}{$\begin{array}{l}\text { Number of prior lines of treatment in } \\
\text { current op medication users }(n=422\end{array}$} \\
\hline & & Hip $(n=145)$ & Spine $(n=178)$ & Current & Previous & 0 & 1 & 2 or more \\
\hline Any treatment ${ }^{\mathrm{a}}$ & $626(16.5 \%)$ & $52(35.9 \%)$ & $103(57.9 \%)$ & $422(67.4 \%)$ & $204(32.6 \%)$ & $321(76.1 \%)$ & $80(19.0 \%)$ & $21(5.0 \%)$ \\
\hline Denosumab $^{b}$ & $187(29.9)$ & $19(36.5)$ & $48(46.6)$ & $174(41.2)$ & $13(6.4)$ & $91(28.3)$ & $61(76.3)$ & $18(85.7)$ \\
\hline Oral $\mathrm{BP}^{\mathrm{b}}$ & $422(67.4)$ & $32(61.5)$ & $61(59.2)$ & $269(63.7)$ & $153(75.0)$ & $187(58.3)$ & $6(7.5)$ & $1(4.8)$ \\
\hline Parenteral $\mathrm{BP}^{\mathrm{b}}$ & $85(13.6)$ & $10(19.2)$ & $18(17.5)$ & $57(13.5)$ & $28(13.7)$ & $27(8.4)$ & $8(10.0)$ & $1(4.8)$ \\
\hline $\mathrm{PTH}^{\mathrm{b}}$ & $8(1.3)$ & $1(1.9)$ & $3(2.9)$ & $4(0.9)$ & $4(2.0)$ & $1(0.3)$ & $1(1.3)$ & $1(4.8)$ \\
\hline SERM $^{\mathrm{b}}$ & $41(6.5)$ & $4(7.7)$ & $6(5.8)$ & $28(6.6)$ & $13(6.4)$ & $16(5.0)$ & $4(5.0)$ & $0(0.0)$ \\
\hline Strontium $^{\mathrm{b}}$ & $40(6.4)$ & $4(7.7)$ & $0(0.0)$ & $21(5.0)$ & $19(9.3)$ & $4(1.2)$ & $1(1.3)$ & $0(0.0)$ \\
\hline
\end{tabular}

$B P$, bisphosphonate; $O P$, osteoporosis; $P T H$, parathyroid hormone; $R A N K L$, receptor activator of nuclear factor kappa-B ligand; SERM, selective estrogen receptor modulators

${ }^{\text {a }}$ Proportions are expressed as a percentage of the total patients in that category ( $n$ in the header)

${ }^{\mathrm{b}}$ The proportion of patients with an individual OP treatment is expressed as a percentage of patients with any treatment in that column

to be a key factor in the decision to treat patients at increased risk of fracture. It is unclear why approximately one-third of patients with a diagnosis still remain untreated: physicians may be managing multiple other competing conditions and may consider vitamin $\mathrm{D} /$ calcium supplements sufficient to reduce fracture risk, or patients may have declined treatment because of confusion about the future risk of fracture compared with the risk of adverse effects, or may have developed side effects to one treatment and not transitioned to another.

Our data demonstrate that oral bisphosphonates are still the most widely used agents, comprising $58 \%$ of first-line OP medication $(58.3 \%$ ) compared with $28 \%$ receiving denosumab, and only $8 \%$ accessing parenteral bisphosphonate therapy. The vast majority of patients on second-line therapy received non-oral treatment (denosumab, $76 \%$; parenteral BP, $10 \%$ ), a pattern that is consistent with recommendations in key European guidelines $[9,14-16]$.

This study has a number of strengths as well as limitations. Care was taken during the study design and implementation to reduce bias and optimize the generalizability of the results within the participating countries. For example, site selection and assessment was conducted by a third party, to avoid inclusion of sites with an existing relationship with the sponsor and potentially having a particular interest in OP. The prevalence of risk factors and comorbidities is consistent with other studies [17-19], indicating that the current study population is representative of the primary care population. Furthermore, the consistency of patient characteristics between those recruited early to the study and those recruited later indicates that the selection criteria were not altered to fulfill enrollment targets. Our findings are consistent with a recent study in older Swedish women that showed a treatment gap of $78 \%$ in those eligible for treatment according to national guidelines [20]. Although there appears to be a clear link between osteoporosis diagnosis and the treatment gap, the study was not designed to establish causality for factors associated with lack of treatment. We cannot exclude that a treatment decision by a GP may affect whether they enter an osteoporosis diagnosis into the medical record. Notably, the treatment gap was lowest in the subgroup of patients with a T-score $\leq-2.5(21 \%)$, a finding that suggests that treatment followed the diagnosis at least in this subset of patients.

Taken together, our study and earlier studies suggest no improvement in treatment rates over the last 10 years. The increase in the treatment gap could be attributed to several factors such as misconceptions about OP, lack of perceived benefits of therapy, concern about side effects and medication costs, lack of motivation, and lack of patient education [21]. Importantly, inadequate or incorrect information in the media related to OP and its treatment are also associated with a low awareness of the disease and, subsequently, low treatment rates [22]. It is likely that novel strategies will be needed to narrow the treatment gap, particularly in older women. Greater awareness and ease of access to fracture risk assessment and treatment are likely to play a part; for example, the establishment of fracture liaison services in primary or secondary care settings increases BMD uptake and treatment exposure and is associated with reductions in fracture risk [23]. The application of fracture risk assessment in primary care and community settings was the focus of three recent randomized controlled trials of screening using the FRAX tool [17, 24, 25]. A meta-analysis of the three studies [26] showed a statistically significant reduction of major osteoporotic fractures (hazard ratio $[\mathrm{HR}]=0.91 ; 95 \% \mathrm{CI}=0.84-0.98$ ) and hip fractures $(\mathrm{HR}=0.80 ; 95 \% \mathrm{CI}=0.71-0.91)$. The number needed to be treated to prevent one fracture was $25-28$ patients. Educational and information technology-based solutions that could further facilitate fracture risk assessment in primary care could therefore be a critical step; it is of interest that there is a 
negative correlation between frequency of FRAX usage in the 8 countries studied (derived from [27] and the observed treatment gap $(r=-0.74$, data not shown)), though this is not evidence of causation.

In conclusion, our study provides insight into the treatment gap and treatment practices in women $\geq 70$ years of age in the primary care setting in Europe. Improved identification and assessment of fracture risk by primary care physicians should be a priority for medical bodies and policymakers, to enable informed treatment decisions that target those at greatest risk of fracture, and who are most likely to benefit from OP medications.

Acknowledgments Medical writing support was provided by Deepali Mittal, an employee of Bridge Medical Consulting Ltd, and funded by Amgen.

Funding information This study was sponsored by Amgen.

\section{Compliance with ethical standards}

The study has been approved by the appropriate institutional and/or national research ethics committee and has been performed in accordance with the ethical standards as laid down in the 1964 Declaration of Helsinki and its later amendments or comparable ethical standards.

Informed consent was obtained from all individual participants included in the study.

Conflicts of interest Eugene McCloskey: grants/research support from ActiveSignal, Amgen, AR UK, AstraZeneca, Consilient Healthcare, GSK, Hologic, I3 Innovus, Internis, IOF, Lilly, Medtronic, Merck, MRC, Novartis, Pfizer, Roche, Sanofi-Aventis, Servier, Synexus, Tethys, UCB, Unilever, and Warner Chilcott; consultant for ActiveSignal, Amgen, Consilient Healthcare, Fresenius Kabi, GSK, Synexus, and UCB; speaking fees from Agnovos, Amgen, AstraZeneca, Consilient Healthcare, GSK, Hologic, Internis, Lilly, Pfizer, Roche, Servier, and UCB. Bernard Cortet has received fees for occasional interventions as an expert or speaker from Alexion, Amgen, Expanscience, Ferring, Lilly, Medtronic, MSD, Mylan, Novartis, Roche Diagnostics, and UCB. E. Czerwinski: traveling grant from Amgen. Kerry Palmer, Robert Stad, James O'Kelly: Amgen employment and stock ownership. Socrates Papapoulos: consulting/speaking fees from Amgen, Axsome, Gador, Radius Health, UCB. Peyman Hadji: grants/ research, consultancy, and lecture fees from Amgen, AstraZeneca, Dr. Kade/Besins, Exeltis, Gedeon Richter, Hexal, Hologic, Lilly, Mylan, Novartis, Pfizer, and UCB. Jeetandera Rathi, Stephane Heijmans, Mark Blagden, Juraj Payer: no disclosures.

Open Access This article is licensed under a Creative Commons Attribution-NonCommercial 4.0 International License, which permits any non-commercial use, sharing, adaptation, distribution and reproduction in any medium or format, as long as you give appropriate credit to the original author(s) and the source, provide a link to the Creative Commons licence, and indicate if changes were made. The images or other third party material in this article are included in the article's Creative Commons licence, unless indicated otherwise in a credit line to the material. If material is not included in the article's Creative Commons licence and your intended use is not permitted by statutory regulation or exceeds the permitted use, you will need to obtain permission directly from the copyright holder. To view a copy of this licence, visit http://creativecommons.org/licenses/by-nc/4.0/.

\section{References}

1. Ström O, Borgström F, Kanis JA et al (2011) Osteoporosis: burden, health care provision and opportunities in the EU. A report prepared in collaboration with the International Osteoporosis Foundation (IOF) and the European Federation of Pharmaceutical Industry Associations (EFPIA). Arch Osteoporos 6:59-155

2. Kanis JA, Cooper C, Rizzoli R et al (2017) Identification and management of patients at increased risk of osteoporotic fracture: outcomes of an ESCEO expert consensus meeting. Osteoporos Int 28: 2023-2034

3. Hernlund E, Svedbom A, Ivergård M et al (2013) Osteoporosis in the European Union: medical management, epidemiology and economic burden. A report prepared in collaboration with the International Osteoporosis Foundation (IOF) and the European Federation of Pharmaceutical Industry Associations (EFPIA). Arch Osteoporos 8:136

4. Compston JE, McClung MR, Leslie WD (2019) Osteoporosis. Lancet 393:364-376

5. Chenot R, Scheidt-Nave C, Gabler S, Kochen MM, Himmel W (2007) German primary care doctors' awareness of osteoporosis and knowledge of national guidelines. Exp Clin Endocrinol Diabetes 115:584-589

6. Lespessailles E, Cotté F-E, Roux C, Fardellone P, Mercier F, Gaudin A-F (2009) Prevalence and features of osteoporosis in the French general population: the Instant study. Joint Bone Spine 76: $394 \mathrm{e} 400$

7. Sahota O, Worley A, Hosking J (2000) An audit of current clinical practice in the management of osteoporosis in Nottingham. Journal of Public Health Medicine 22:466-472

8. Judge A, Javaid MK, Leal J, et al (2016) Chapter 7: Primary care and hospital care costs for hip fracture patients. In: Models of care for the delivery of secondary fracture prevention after hip fracture: a health service cost, clinical outcomes and cost-effectiveness study within a region of England. NIHR Journals Library, Southampton, UK. Health Services and Delivery Research;4.28. https://www. ncbi.nlm.nih.gov/books/NBK385602/. Accessed Sept 20, 2019

9. Compston J, Cooper A, Cooper C et al (2017) UK clinical guideline for the prevention and treatment of osteoporosis. Arch Osteoporos 12:43. https://doi.org/10.1007/s11657-017-0324-5 Accessed 17 Sept 2019

10. Marsh D, Åkesson K, Beaton DE (2011) Coordinator-based systems for secondary prevention in fragility fracture patients. Osteoporos Int 22:2051-2065

11. Kanis JA, Svedbom A, Harvey N et al (2014) The osteoporosis treatment gap. J Bone Miner Res 29:1926-1928

12. McCloskey E, Rathi J, Heijmans S et al (2019) Osteoporosis (OP) diagnosis and treatment of women aged $\geq 70$ years in primary care: results from a large European cross-sectional study. World Congress on Osteoporosis, Osteoarthritis and Musculoskeletal Diseases, Paris, France, April 4-7, (poster P997)

13. Díez-Pérez A, Hooven FH, Adachi JD et al (2011) Regional differences in treatment for osteoporosis. The Global Longitudinal Study of Osteoporosis in Women (GLOW). Bone 49:493-498

14. Dachverband Osteologie. DVO-LEITLINIE 2017 zur Prophylaxe, Diagnostik und Therapie der Osteoporose bei postmenopausalen Frauen und bei Männern (German). https://www.dv-osteologie. org/uploads/Leitlinie\%202017/DVO\%20Leitlinie_ Kitteltaschenversion_gesamt.pdf. Accessed 13 Mar 2020.

15. Briot K, Roux C, Thomas T, Blain H et al (2018) 2018 update of French recommendations on the management of postmenopausal osteoporosis. Joint Bone Spine 85:519-530 
16. Kanis JA, Cooper C, Rizzoli R et al (2019) European guidance for the diagnosis and management of osteoporosis in postmenopausal women. Osteoporosis Int 30:3-44

17. Shepstone L, Lenaghan E, Cooper C et al (2018) for SCOOP Study Team. Screening in the community to reduce fractures in older women (SCOOP): a randomised controlled trial. Lancet 391:741-747

18. Dennison EM, Compston JE, Flahive J et al (2012) Effect of comorbidities on fracture risk: findings from the Global Longitudinal Study of Osteoporosis in Women (GLOW). Bone 50:1288-1293

19. Raherison C, Girodet P-O (2009) Epidemiology of COPD. Eur Respir Rev 18:213-221

20. Lorentzon M, Nilsson AG, Johansson H, Kanis JA, Mellström D, Sundh D (2019) Extensive undertreatment of osteoporosis in older Swedish women. Osteoporosis International 30:1297-1305

21. Hiligsmann M, Cornelissen D, Vrijens B et al (2019) Determinants, consequences and potential solutions to poor adherence to antiosteoporosis treatment: results of an expert group meeting organized by the European Society for Clinical and Economic Aspects of Osteoporosis, Osteoarthritis and Musculoskeletal Diseases (ESCEO) and the International Osteoporosis Foundation (IOF). Osteoporos Int 30:2155-2165
22. Cipriani C, Pepe J, Minisola S, Lewiecki EM (2018) Adverse effects of media reports on the treatment of osteoporosis. J Endocrinol Invest 41:1359-1364

23. Wuab CH, Tuc ST, Chang YF et al (2018) Fracture liaison services improve outcomes of patients with osteoporosis-related fractures: a systematic literature review and meta-analysis. Bone 111:92-110

24. Rubin KH, Rothmann MJ, Holmberg T et al (2018) Effectiveness of a two-step population-based osteoporosis screening program using FRAX: the randomized Risk-stratified Osteoporosis Strategy Evaluation (ROSE) study. Osteoporos Int 29:567-578

25. Merlijn T, Swart KM, van Schoor NM et al (2019) The effect of a screening and treatment program for the prevention of fractures in older women: a randomized pragmatic trial. J Bone Miner Res 34: 1993-2000

26. Merlijn T, Swart KMA, van der Horst HE, Netelenbos JC, Elders PJM (2020) Fracture prevention by screening for high fracture risk: a systematic review and meta-analysis. Osteoporos Int 31:251-257

27. Chotiyarnwong P, Harvey NC, Johansson H et al (2019) Temporal changes in access to FRAX(R) in Thailand between 2010 and 2018. Arch Osteoporos 14:66

Publisher's note Springer Nature remains neutral with regard to jurisdictional claims in published maps and institutional affiliations. 